\title{
Dispersion of coagulated nano-particles in high shear field
}

\author{
Hideo Watanabe, Takumi Suzuki, Takeshi Endo, Masayoshi Fuji, Minoru Takahashi \\ Ceramics Research Laboratory, Nagoya Institute of technology, 10-6-29 Asahigaoka, Tajimi, Gifu, 507-0071 Japan \\ Tel: +81-572-27-6811, Fax: 81-572-27-6812, e-mail: h.watanabe@nitech.ac.jp
}

Well dispersion of nano-particles is necessary in order to bring out their unique properties, such as solubility, reactivity, and electromagnetic, optical and mechanical properties. However, it is difficult in general to disperse nano-particles homogeneously in liquid media because they are very prone to coagulate each other. In this study, we have developed and constructed a new apparatus for dispersing particles that basically consists of co-axial rotor and a static vessel to generate a high shear field between them. Coagulated particles are subject to high shear forces which enable to disperse the coagulated particles. In this paper we have firstly designed the dispersing apparatus, and have then carried out the dispersion tests with calcium carbonate particle which has tens nanometer size as a primary particle. The dispersion performance has been evaluated by measuring particle size distribution with taking account of shape of rotors, dimensions of the gap between rotor and vessel, rim speed of the rotor and operating time. It has been found that a smaller gap results in higher dispersing performance since higher velocity gradient is formed in the gap.

Key words: particle dispersion, nano-particles, high shear field, calcium carbonate

\section{INTRODUCTION}

Well dispersion of the nano-particles in liquid or gas media is necessary in order to reveal their unique properties which are owing to their small particle size (high specific surface area) such as solubility, reactivity, and unusual properties of electromagnetism, optics and mechanics different from the bulk. However, it is difficult in general to disperse nano-particles into the primary particles since they are very prone to coagulate each other due to high cohesive forces based on their high specific surface area. Therefore, the dispersion of nano-particles shall be one of the most important key techniques in so-called nano-technology. The method for particle dispersion can be categorized mainly into two approaches as follows: 1) mechanical dispersion methods in which physical energy more than cohesive forces working between particles is put to the coagulated particles to be dispersed, 2) physicochemical dispersion methods in which particle surfaces are subjected to chemical modification and the particle dispersibility would be improved. The former mechanical methods include use of ultrasonic wave illumination [1], media follow $[2,3]$ and so forth. The later physicochemical methods include surface modification treatment using silane coupling agents or alcohols and so forth $[4,5]$.

In this study, we have developed an apparatus for dispersing particles that basically consists of co-axial rotor and a vessel to generate a high shear field between them. Coagulated particles are subject to high shear forces which enable to disperse the coagulated particles. The apparatus is easy to be applied in continuous process and we would intend to combine the apparatus with chemical surface dispersion method in the next phase of our research program. In this paper we have firstly designed the dispersing apparatus, and have then carried out the dispersion tests with calcium carbonate particle which has tens nanometer size as a primary particle. The dispersion performance has been evaluated by measuring particle size distribution in the calcium slurry with taking account of shape of rotors, dimensions of the gap between rotor and vessel, rim speed and treatment time.

\section{EXPERIMENTAL}

2.1 Design of apparatus for particle dispersion with high speed rotational flow

Figure 1 shows a schematic diagram of experimental setup used in this study for particle dispersion with high speed rotational flow. The setup consists of a slurry tank, a pump circulating the slurry and a dispersion vessel which consists of a rotor and a static vessel. The slurry flown from the tank comes into the vessel from the bottom and goes through a gap between a rotor and a vessel and comes out from the top of the vessel and then return into the tank. The slurry tank is filled with nitrogen gas (with slight gas flow) apart from the slurry, and is equipped with a cold water jacket to prevent the slurry from overheating.

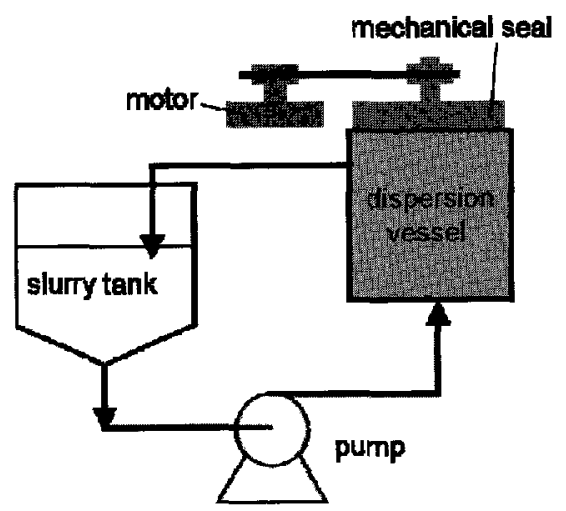

Fig.1 Schematic diagram of experimental setup for dispersing particles in slurry 
The two types of rotor and vessel were designated as shown in figures 2 . The first configuration is the rotor in shape of a headstand cup and the vessel in shape of a cylinder with a pin cylinder at the center of the bottom as shown in Fig.2 (a), this is called "type I" vessel in this paper. The other is a single cylindrical rotor and a vessel as shown in Fig.2 (b), this is called vessel "type II" vessel in this paper. In both the cases, rotor and vessel are in a co-axial position with the center for rotation of the rotor. Major dimensions are shown in Figs.2, and the gap between the rotor and the vessel is $1.0 \mathrm{~mm}$ for type I and 1.0 or $0.5 \mathrm{~mm}$ for type II.

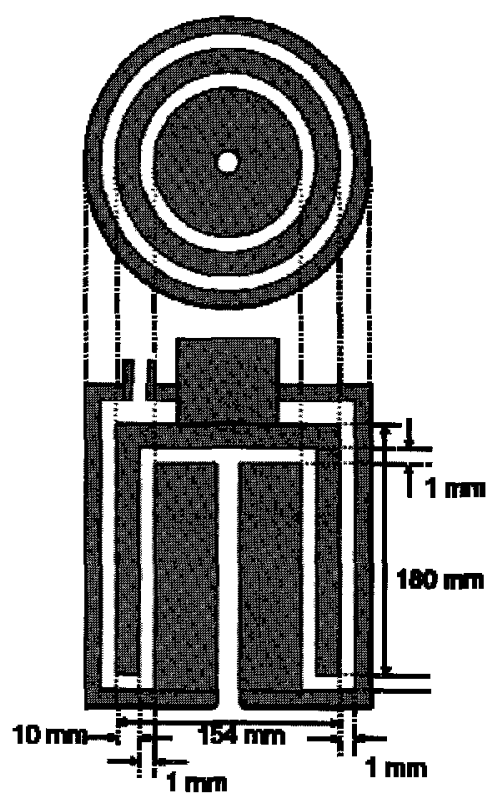

(a) Type I: headstand cup rotor

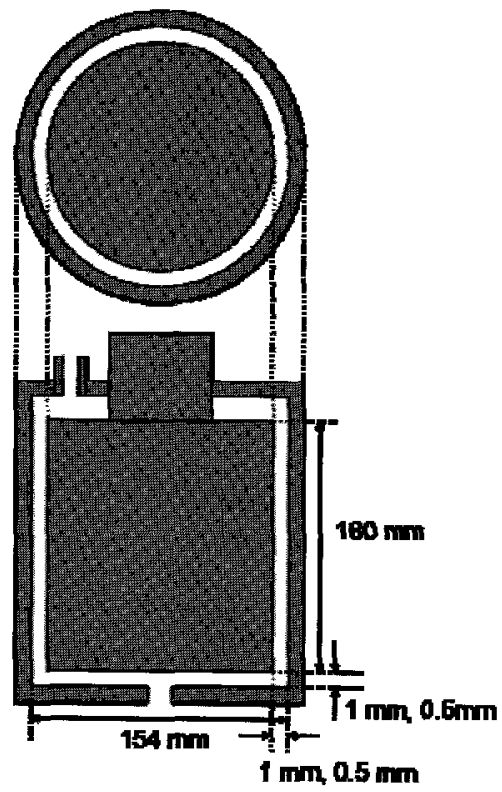

(b) Type II: single cylindrical rotor

Figs.2 Schematic diagrams of rotor and vessel

\subsection{Sample slurries}

Calcium carbonate (average size $60 \mathrm{~nm}$, Nanocube 60 , Nittetsu Mining, Japan) was used as a sample powder, and sodium hexametaphosphate (Kanto Chemical, Japan) was used as a dispersant. The calcium carbonate was mixed with distilled water and was stirred using a magnetic stirrer for $30 \mathrm{~min}$. The solid loading was kept at 4.5 mass \% (1.65 vol.\%) throughout this paper. The amount of dispersant added to the slurry is 1.0 mass $\%$ to the mass of the powder in the slurry.

\subsection{Particle dispersion test}

$2 \mathrm{~L}$ of the calcium carbonate slurry was put into the slurry tank and was circulated with the constant flow rate of $0.5 \mathrm{~L} / \mathrm{min}$. to the dispersion vessel and then return to the tank. The circulation was taken for 10 minutes for all the dispersion tests as a preliminary mixing. The rotor eventually began to rotate with a constant velocity in the range of 21 to $40 \mathrm{~m} / \mathrm{s}$ as rim speed. At certain treatment time elapsed, the slurry was sampled from the tank and particle size distribution of the sample was measured using a laser diffraction method (Microtrac MT3000, Nikkiso, Japan).

\section{Results and discussion}

3.1 Effect of rotor shapes for dispersion performance

Figure 3 shows particle size distributions of slurries without dispersion treatment (original) and with the treatment using either Type I or II (gap $1 \mathrm{~mm}$ ) rotor with the rim speed of $40 \mathrm{~m} / \mathrm{s}$ for 5 minutes. The $y$-axis in this figure indicates frequency for each size fraction in volume percent, and the $\mathrm{x}$-axis indicates particle size in a logarithmic scale. As compared to the original sample of which average size is $21.4 \mu \mathrm{m}$, the treated samples have smaller particle size distributions. The data of Type II shows lower frequency in larger size fractions around $10 \mu \mathrm{m}$, and higher frequency in smaller fractions less then several $\mu \mathrm{m}$ as compared to that of Type I, while the difference is not so significant. The Type I vessel have a longer path than Type II with the same gap of $1.0 \mathrm{~mm}$, so that the result imply that only the gap with outer side of the Type I vessel can contribute to particle dispersion.

\subsection{Effect of gap size for dispersion performance}

Figure 4 shows the effect of gap size for particle dispersion performance. In these cases, Type II vessel was used with the same rim speed of $40 \mathrm{~m} / \mathrm{s}$. The narrower gap $(0.5 \mathrm{~mm})$ obviously results in smaller size distribution as compared to wider gap $(1.0 \mathrm{~mm})$. This is because of velocity gradient of shear flow at the gap increase with decreasing the gap size. In the range of this paper, the gap less than $0.5 \mathrm{~mm}$ has not been tested but would expected to be more efficient for dispersing coagulated particles. 


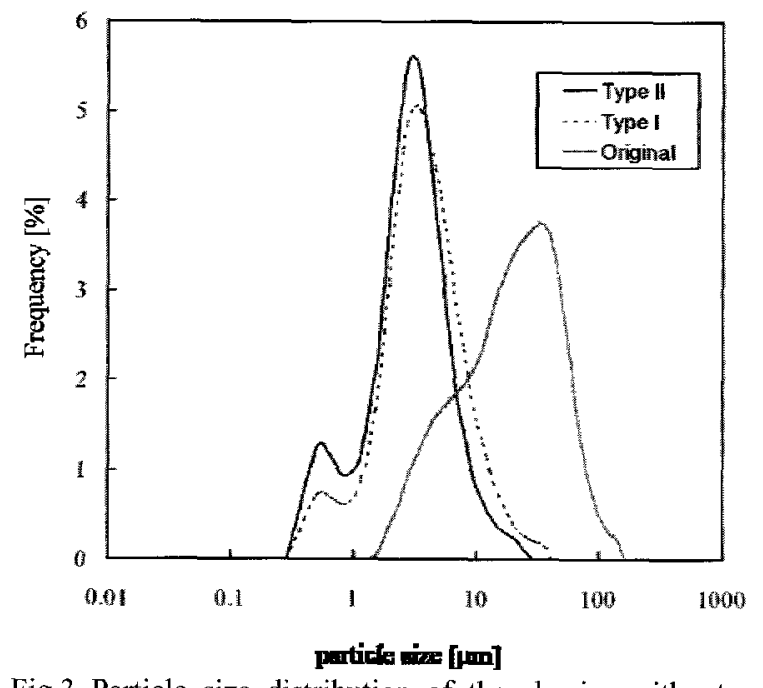

Fig.3 Particle size distribution of the slurries without dispersion treatment (original) and with the treatment using either Type I or II vessel (shown in Figs.2) with rim speed of $40 \mathrm{~m} / \mathrm{s}$. No dispersant was added to the slurries.

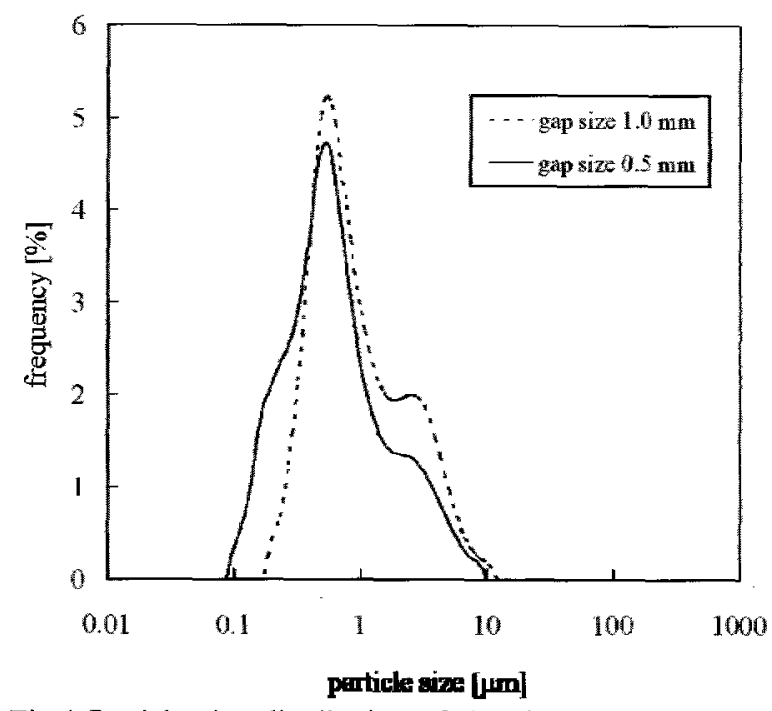

Fig.4 Particle size distribution of the slurries with the dispersion treatment using Type II rotor with different gap sizes of 1.0 or $0.5 \mathrm{~mm}$ (treatment time $15 \mathrm{~min}$, rim speed of $40 \mathrm{~m} / \mathrm{s}$, dispersant was added to the slurries.)

\subsection{Effect of rim speed for dispersion performance}

Figure 5 shows relationship between particle size and rim speed. The y-axis is shown in logarithmic scale. The keys in this figure correspond to the particle size at which cumulative undersize ratio is $10 \%(\mathrm{~d} 10), 50 \%$ (d50) or $90 \%(\mathrm{~d} 90)$. The particle sizes decrease with increasing the rim speed. The decrease in $\mathrm{d} 10$ for high rim speed $(25-40 \mathrm{~m} / \mathrm{s})$ is slighter than that of d50 and d90. This might be because larger coagulated particles are easier to be dispersed but smaller particles especially in submicron size are harder to be dispersed.

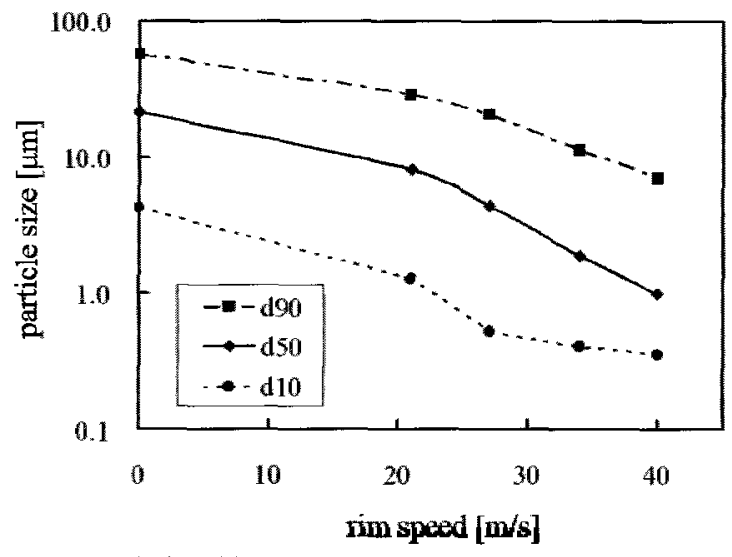

Fig.5 Relationship between particle sizes with cumulative undersize ratio of $10 \%(\mathrm{~d} 10), 50 \%(\mathrm{~d} 50)$ and $90 \%$ (d90) in volume and rim speed of the Type II vessel with gap size $0.5 \mathrm{~mm}$ (treatment time $5 \mathrm{~min}$. dispersant was added to the slurries.)

3.4 Effect of treatment time for dispersion performance

Figure 6 shows time course of the particle sizes. The particle size decrease considerably by 5 minutes treatment, and gradually for the time 10 to 15 minutes The 5 minutes is roughly equal a time required for all slurry input in the tank to come into the dispersion vessel and come back to the tank. Therefore the result can be interpreted that the only one-time dispersion treatment, which means one-time passing through the reaction vessel, can reduce the particle size in the level of the lowest limit that is attainable by this apparatus developed in this study.

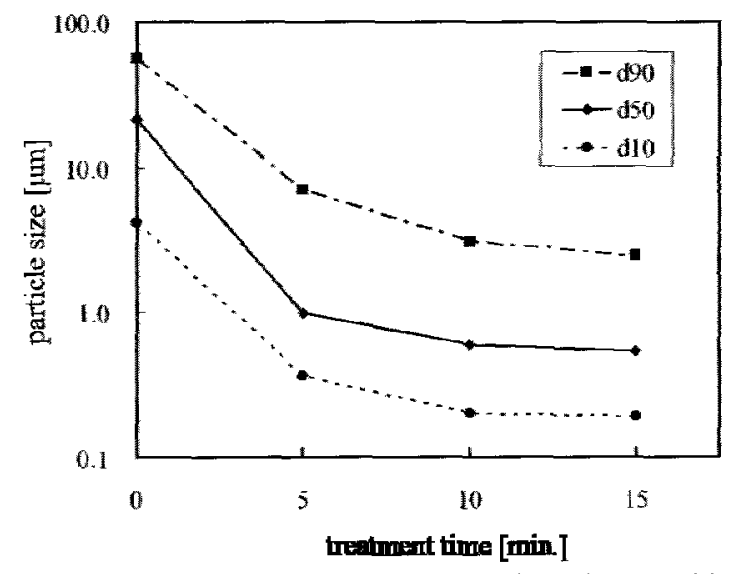

Fig.6 Relationship between particle sizes with cumulative undersize ratio of $10 \%$ (d10), 50\% (d50), and $90 \%(\mathrm{~d} 90)$ in volume and treatment time (rim speed $40 \mathrm{~m} / \mathrm{s}$, dispersant was added to the slurries.)

Even after dispersing treatment with rim speed $40 \mathrm{~m} / \mathrm{s}$ for 15 minutes, the particle size is $540 \mathrm{~nm}$ as d50 at 15 min in Fig. 6 and is much larger than the primary particle size $(60 \mathrm{~nm})$. This might be attributed to re-coagulation which might occur immediately after dispersion treatment. Therefore we have been planning that chemical surface modification to the particles using 
a coupling agent is carried out in the high shear field, which would enable to enhance dispersibility of the dispersed particles and hence to prevent from re-coagulating them. This will be published elsewhere [6].

\section{Conclusions}

We have developed and constructed a new apparatus for dispersing particles that basically consists of co-axial rotor and a static vessel to generate a high shear field between them. The dispersion tests with calcium carbonate particle which has tens nanometer size as a primary particle have been carried out. The dispersion performance has been evaluated by measuring particle size distribution with taking account of shape of rotors, dimensions of the gap between rotor and vessel, rim speed of the rotor and operating time. It has been found that a smaller gap results in higher dispersing performance since higher velocity gradient is formed in the gap.
Acknowledgements

A part of the present work has been supported by a grant from the METI (Ministry of Economy Trade and Industry) from 2004 to 2006 as a part of the Industrial Science and Technology Research and Development "Research and Development of the Ultra Low Dielectric Insulation Film and Anticorrosive Film using Hollow Nanoparticles". One of the members joining the project above, Chuo Kakoki Co. Ltd. is acknowledged for their contributions to constructing the apparatus used in this study.

\section{References}

[1] K. Higashitani, K. Yoshida, N. Tanise and H. Murata, Colloids and Surfaces A, 81, 167-175 (1993).

[2] Ko Higashitani, Nobufumi Inada and Toyohiko Ochi, Colloids and Surfaces, 56, 13-23 (1991).

[3] K. Higashitani, N. Tanie, A. Yoshiba and A. Kondo, J. Chem. Eng. Japan, 25, 502-507 (1992)

[4] M. Fuji, S. Ueno, T. Watanabe and M. Chikazawa, Colloid Polym. Sci., 278, 30-36 (2000)

[5] C. Takai, M. Fuji and M. Takahashi, Colloids and Surfaces A, 292, 79-82 (2007).

[6] H. Watanabe, T. Suzuki, T. Endo, M. Fuji and M. Takahashi, to be submitted for J. Colloid Interface Sci. 